ARTICLE

Received 31 Jan 2013 | Accepted 20 Feb 2013 | Published 27 Mar $2013 \quad$ DOl: 10.1038/ncomms2636

\title{
CAND1 controls in vivo dynamics of the cullin 1-RING ubiquitin ligase repertoire
}

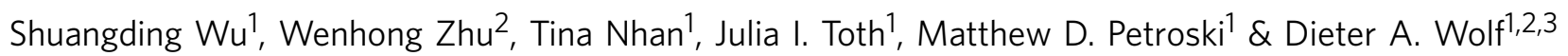

The combinatorial architecture of cullin 1-RING ubiquitin ligases, in which multiple F-box containing substrate receptors compete for access to CUL1, poses special challenges to assembling cullin 1-RING ubiquitin ligase complexes through high affinity protein interactions while maintaining the flexibility to dynamically sample the entire F-box containing substrate receptor repertoire. Here, using highly quantitative mass spectrometry, we demonstrate that this problem is addressed by CAND1, a factor that controls the dynamics of the global cullin 1-RING ubiquitin ligase network by promoting the assembly of newly synthesized F-box containing substrate receptors with CUL1-RBX1 core complexes. Our studies of in vivo cullin 1-RING ubiquitin ligase dynamics and in vitro biochemical findings showing that CAND1 can displace F-box containing substrate receptors from Cul1p suggest that CAND1 functions in a cycle that serves to exchange F-box containing substrate receptors on CUL1 cores. We propose that this cycle assures comprehensive sampling of the entire F-box containing substrate receptor repertoire in order to maintain the cullin 1-RING ubiquitin ligase landscape, a function that we show to be critical for substrate degradation and normal physiology.

\footnotetext{
${ }^{1}$ Signal Transduction Program, Sanford-Burnham Medical Research Institute, La Jolla, California 92037, USA. ${ }^{2} \mathrm{NCl}$ Cancer Center Proteomics Facility, Sanford-Burnham Medical Research Institute, La Jolla, California 92037, USA. ${ }^{3}$ San Diego Center for Systems Biology, Sanford-Burnham Medical Research Institute, La Jolla, California 92037, USA. Correspondence and requests for materials should be addressed to D.A.W. (email: dwolf@sanfordburnham.org).
} 
C ullin 1-RING ubiquitin ligases (CRL1s) are multifunctional ubiquitin ligases that uniquely exploit combinatorial diversity in order to achieve unparalleled versatility in substrate targeting and control of cell physiology ${ }^{1-3}$. This combinatorial layout where multiple F-box containing substrate receptors (FBPs) compete for access to CUL1 poses special challenges to assembling CRL1 complexes through high affinity protein interactions while maintaining the flexibility to dynamically sample the entire FBP repertoire. Mounting evidence has implicated mechanisms related to the reversible modification of CUL1 with the ubiquitin-related peptide NEDD8 in this regulation ${ }^{4}$, but no definitive model has been substantiated experimentally.

Conjugation of NEDD8 to CUL1 stimulates the ubiquitin ligase activity of CRL1s $\mathrm{s}^{5,6}$, and deneddylation by the COP9 signalosome resets CRL1s into an inactive state ${ }^{7-9}$. Deneddylation has two important consequences: it prevents the autocatalytic destruction of FBPs ${ }^{10-12}$ and it allows CUL1 to associate with CAND1, a highly conserved protein that inhibits CUL1 neddylation and hence CRL1 activity in vitro ${ }^{13-18}$. This inhibition can be overcome by purified SKP1-FBP heterodimers, which dissociate the CUL1CAND1 complex in vitro ${ }^{18,19}$. Paradoxically, however, CAND1 was also shown to promote CRL function in vivo ${ }^{12,14,20-23}$.

In pursuit of this paradox, we previously showed that fission yeast cells deleted for $k n d 1$, the orthologue of human CAND1, display an imbalance in CRL complexes formed between Cullp and two epitope-tagged FBPs, Poflp-Myc and Pof $3 p-M_{y c}{ }^{12}$. Whereas CRL1 1 Poflp-Myc complexes increased, CRL1 1 Pof3p-Myc complexes were depleted in cells lacking $k n d 1$. The $\sim 2$-fold decrease in the CRL1 ${ }^{\text {Pof3p-Myc }}$ complex was sufficient to cause phenotypic defects that mirrored a $\Delta$ pof 3 deletion mutant ${ }^{12,24}$, a finding that reinforced a positive role of CAND1/Knd1p in CRL1 control. Similar imbalances were reported for CUL1-TIR1 interactions in A. thaliana and for CUL3-KEAP1 interactions in human cells ${ }^{25,26}$. The latter studies also demonstrated that substrate degradation by CRL1 $1^{\text {TIR1 and }}$ CRL $3^{\text {KEAP1 is }}$ compromised either in the absence of CAND1 or when CAND1 is overexpressed. Based on these findings, we proposed that a CAND1-mediated cycle of CRL1 complex disassembly and subsequent reassembly maintains the cellular balance of CRL1 complexes and optimal CRL1 activity ${ }^{12}$. However, a subsequent study using siRNA-mediated knockdown in human cells achieved a partial reduction in CUL1-CAND1 complex but observed no significant effect on the recruitment of SKP1 (and presumably FBPs) to CUL1 and, therefore, relinquished a role of CAND1 in CRL1 assembly and remodelling ${ }^{27}$.

We have used highly quantitative mass spectrometry to rigorously assess the impact of complete genetic depletion of CAND1/Knd1p on the global CRL1 repertoire and its assembly/disassembly dynamics. We demonstrate that CAND1/Kndlp has a crucial role in maintaining a balanced repertoire through mechanisms that are consistent with our previously proposed CAND1 cycle ${ }^{12}$.

\section{Results}

CAND1/Knd1p maintains the global CRL1 repertoire. To test the effect of complete genetic ablation of CAND1 on the native CRL1 repertoire, we immunopurified Cullp-associated proteins from wild-type and $\Delta k n d 1$ fission yeast cells differentially labelled with stable isotopes ${ }^{28}$ and quantified them by liquid chromatography and tandem mass spectrometry (LC-MS/MS). Whereas $\Delta k n d 1$ cells were grown in medium containing regular ammonium- ${ }^{14} \mathrm{~N}$ chloride as the nitrogen source, the wild-type cells were metabolically labelled with ammonium $-{ }^{15} \mathrm{~N}$ chloride with an efficiency of $>98 \%$. Cultures were mixed at a ratio of 1:1 and processed as a single sample for lysate preparation, Cullp immunopurification and LC-MS/MS to quantify the relative abundance of Cullp-associated proteins in wild-type and $\Delta k n d 1$ cells based on averaged ${ }^{14} \mathrm{~N} /{ }^{15} \mathrm{~N}$ peptide ratios (Fig. 1a). Triplicate experiments revealed statistically significant $(P \leq 0.05)$ differences in Cullp occupancy by various FBPs. While occupancy by Pof1p, Pof7p, Pof9p, Pof10p and Pof14p increased by 1.3 - to 2.2-fold, occupancy by Pof5p, Pof11p, Pof15p and Pop1p decreased by factors of 1.5- to 5-fold (Fig. 1b; Supplementary Table S1; Supplementary Data 1). The modest amplitude of these changes is explained by the fact that $\sim 50 \%$ of Cullp is neddylated in cells ${ }^{10}$. As neddylated Cullp cannot interact with CAND1, this fraction of CRL1 complexes is not responsive to the cellular CAND1 status.

To corroborate these data, we performed a control experiment to exclude that CRL1 complexes rearrange during the immunopurification from mixed ${ }^{14} \mathrm{~N}$ and ${ }^{15} \mathrm{~N}$ cell lysates. For this, we mixed equal parts of ${ }^{15} \mathrm{~N}$-labelled lysate from wild-type cells carrying a Cullp-Myc allele with unlabelled lysate of $\Delta k n d 1$ cells lacking a Cullp-Myc allele. When Cullp complexes were purified and analysed by LC-MS/MS, CRL1 components were almost exclusively represented by ${ }^{15} \mathrm{~N}$ peptides (Supplementary Fig. S1a,b). This finding indicates that ${ }^{14} \mathrm{~N}$-labelled subunits present in the lysate of untagged cells did not enter CRL1 complexes at an appreciable rate after cell lysis. In contrast, proteins that were unspecifically retrieved on the beads were represented by both ${ }^{14} \mathrm{~N}$ and ${ }^{15} \mathrm{~N}$ peptides at roughly equal parts (Supplementary Fig. S1b).

We subsequently applied LC-MS/MS-based absolute protein quantification technology using isotopically labelled reference peptides (absolute quantification (AQUA) ${ }^{29}$ to measure the exact amounts of FBPs retrieved in CRL1 complexes. In agreement with the relative quantification data, the absolute amounts of Pof1p and Pof10p were 1.5-1.7-fold higher in complexes isolated from $\Delta k n d 1$ cells than from wild-type cells (Fig. 1c; Supplementary Data 2). While 3.5\% of Cullp was occupied by Poflp in wild-type cells, the fraction increased to $6.2 \%$ in $\Delta k n d 1$ cells (Supplementary Data 2). Likewise, Cul1p occupancy by Pof $10 p$ increased from $14.5 \%$ to $24 \%$ in $\Delta k n d 1$ cells. These increases were confirmed by reciprocal immunoprecipitation/immunoblotting experiments (Fig. 1d; Supplementary Fig. S1c). Conversely, Pof15p levels were 3.5 -fold lower in Cullp complexes isolated from $\Delta k n d 1$ cells (Fig. 1c) and the fractional occupancy for this FBP was reduced from $4.6 \%$ to $1.5 \%$ (Supplementary Data 2). Again these absolute measurements confirmed the relative quantifications (Fig. 1b).

The observed changes in Cullp-FBP interactions are not due to concordant changes in steady-state FBP levels in $\Delta k n d 1$ cells (Fig. 2a; ref. 12). Likewise, CRL1 core subunits Cullp and Rbxlp were retrieved in equal amounts from both wild-type and $\Delta k n d 1$ cells (Fig. 1b). Skp1p protein levels were $\sim 40 \%$ higher in the mutant as determined by immunoblotting and quantitative mass spectrometry (Fig. 2a,b). Nevertheless, binding of Skp1p to Cullp was decreased by $\sim 25 \%$ in $\Delta k n d 1$ cells (Fig. 1b). This finding, which contrasts with previous measurements in human cells showing that binding of SKP1 to CUL1 is slightly increased upon acute knockdown of $\mathrm{CAND} 1^{13,14,27}$, was highly reproducible across 179 individual Skp1p peptide measurements by LC-MS/MS (Supplementary Data 3 ). It, therefore, appears that Schizosaccharomyces pombe cells devoid of Knd1p activity might have a $\sim 25 \%$ overall reduction in fully assembled CRL1 complexes. The complexes that are assembled, however, dramatically differ in FBP composition relative to wild-type cells.

CAND1/Knd1p regulates CRL1 dynamics. To determine how the CRL1 repertoire of cells lacking CAND1/Knd1p could be 
a

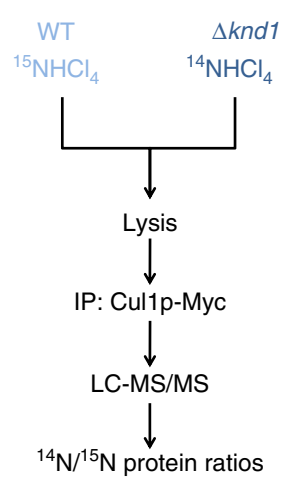

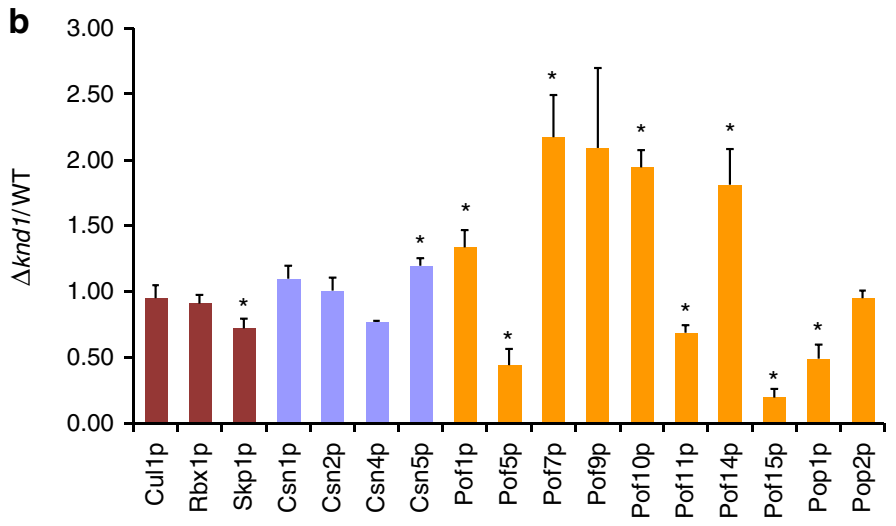

d

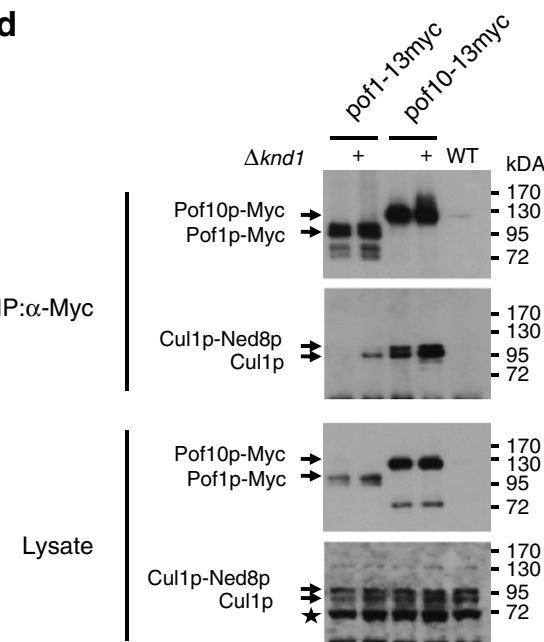

Figure 1 | Effect of Knd1p on the CRL1 repertoire. (a) Wild-type (WT) S. pombe cells and cells deleted for knd1 ( $\Delta$ knd1), which also carry a Myc-tagged allele of endogenous Cul1p, were grown in medium containing either ammonium- ${ }^{14} \mathrm{~N}$ chloride or ammonium- ${ }^{15} \mathrm{~N}$ chloride as the nitrogen sources. Equal numbers of cells were mixed at a ratio of 1:1, followed by the isolation of CRL1 complexes through immunopurification (IP) of Cul1p-Myc. The purified material was analysed by LC-MS/MS to identify Cul1p-associated proteins and to quantify their relative abundance in WT and $\Delta k n d 1$ samples based on ${ }^{14} \mathrm{~N} /{ }^{15} \mathrm{~N}$ peptide/protein ratios. (b) Relative abundance of Cul1p and Cul1p-interacting proteins in WT versus $\Delta k n d 1$ cells. Triplicate data sets (Supplementary Data File 1) were averaged and s.d. values are indicated. Statistically significant changes $(t$-test, $P \leq 0.05)$ are indicated by asterisks. (c) Absolute amounts of Cul1p, Pof1p and Pof10p retrieved from a 1:1 mixture of ${ }^{15} \mathrm{~N}$-labelled WT and ${ }^{14} \mathrm{~N}$-labelled $\Delta$ knd 1 cells by Cul1p IP and mass spectrometry-based quantification by selected ion monitoring. The right hand panel shows the same measurements for Cul1p and Pof15p from an independent Cul1p IP sample. (d) Lysate of WT and $\Delta$ knd1 cells expressing Pof1p or Pof10p tagged with the c-Myc epitope were used in immunoprecipitation with anti-Myc antibodies, followed by detection of co-precipitated proteins with either anti-Myc or anti-Cul1p antibodies. The neddylated and unneddylated forms of Cul1p are indicated. Total cell lysate is shown in the bottom two panels. The asterisk denotes a crossreactivity of the anti-Cul1p antibody.

disturbed in the presence of stable amounts of core components and FBPs, we developed a metabolic ${ }^{15} \mathrm{~N}$ pulse-labelling assay that allowed us to assess CRL1 complex dynamics. Wild-type and $\Delta k n d 1$ cells were maintained in ${ }^{14} \mathrm{~N}$ medium and then switched to ${ }^{15} \mathrm{~N}$ medium for $120 \mathrm{~min}$, followed by lysate preparation, Cullp immunopurification and LC-MS/MS analysis of the retrieved proteins (Fig. 2c). As we had previously shown that CAND1/Knd1p does not affect FBP stability ${ }^{12}$, changes in the ${ }^{14} \mathrm{~N} /{ }^{15} \mathrm{~N}$ peptide ratios of CRL1 components inform primarily on the rate with which newly synthesized components become assembled into CRL1 complexes in wildtype cells relative to $\Delta k n d 1$ cells. Performing this experiment in triplicate, we found a statistically significant diminution in the incorporation of Skp1p and several FBPs into CRL1 complexes (Fig. 2d, Supplementary Data 4). The observation was confirmed in an independent experiment in which the pulse labelling was performed for $240 \mathrm{~min}$ (Supplementary Fig. S2; Supplementary Data 5).
Slow incorporation of newly synthesized FBP may occur because pre-existing CRL1 complexes are stabilized in the absence of knd1. We tested this prediction in an in vivo competition experiment. We perturbed steady-state CRL1 complexes by expressing from a plasmid Myc epitope-tagged Pof15p. This FBP is severely depleted from CRL complexes in $\Delta k n d 1$ cells (Fig. 1b, Supplementary Table S1), indicating that it requires Knd1p to successfully compete for CRL1 core subunits. Pof15p was expressed in a strain that harbours endogenously tagged Pof10p-Myc, an FPB that strongly accumulates in CRL1 complexes in $\Delta k n d 1$ cells (Fig. $1 \mathrm{~b}$, Supplementary Table S1). The strains were constructed in two backgrounds, wild-type and $\Delta k n d 1$. Myc-Pof15p expression was turned on and CRL1 complexes were immunoprecipitated after different times and monitored for the levels of co-precipitated Pof10p-Myc. We found that a $\sim 10$-fold excess of exogenous Pof15p efficiently displaced Pof10p-Myc from Cullp (Fig. 3a). This competition was completely abolished by disrupting the 
a

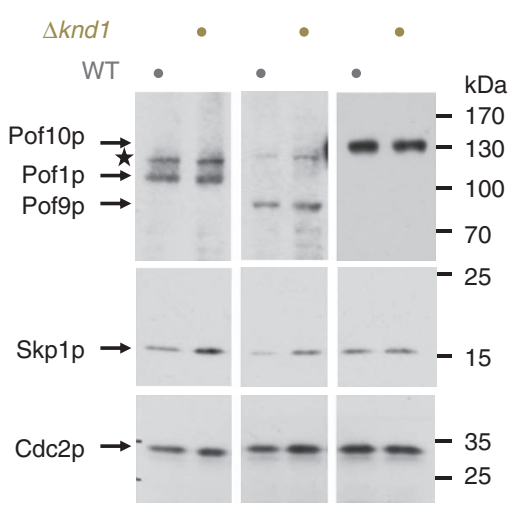

C

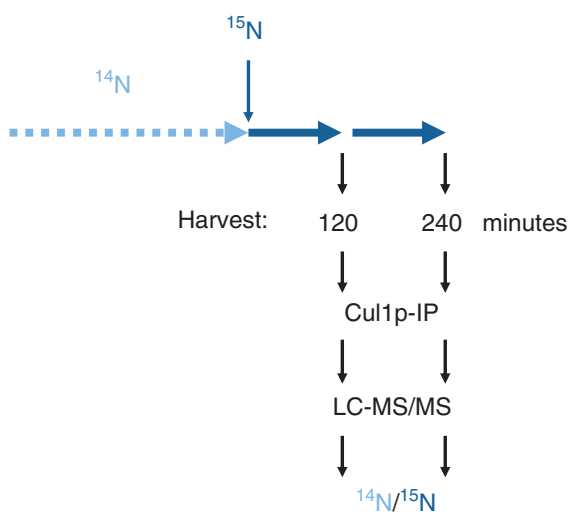

b

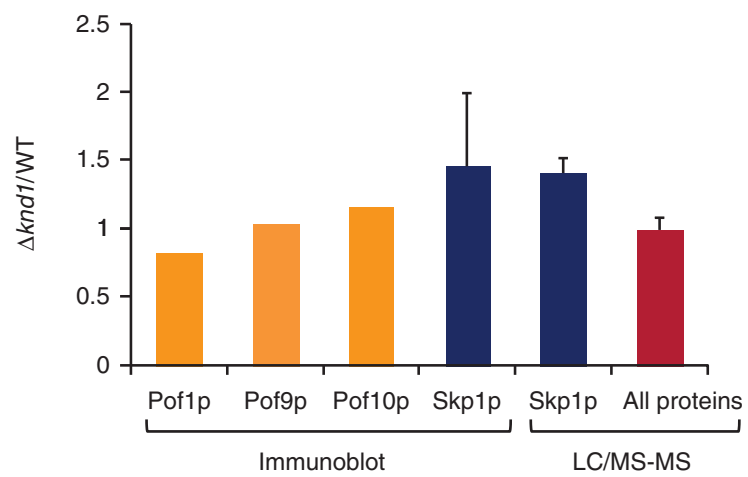

d

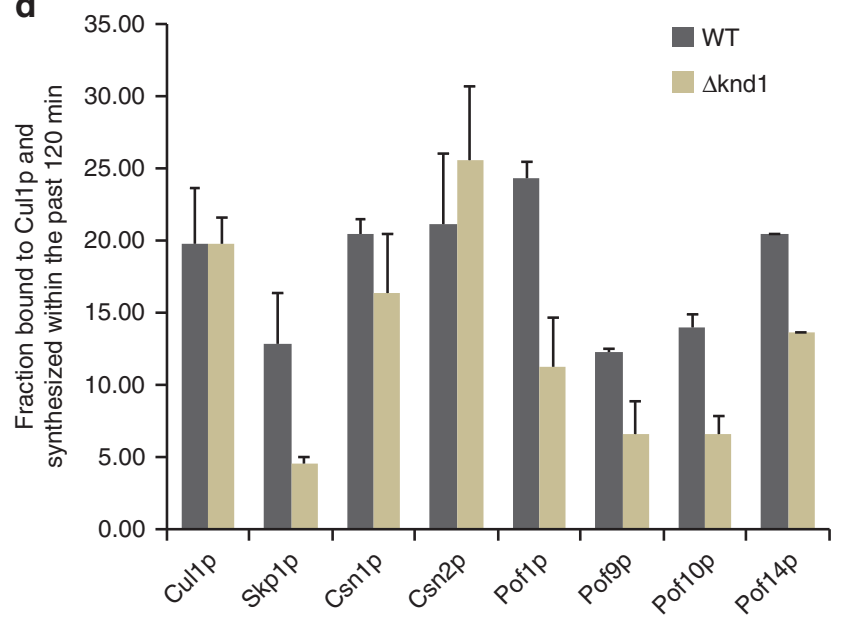

Figure 2 | Effect of Knd1p on CRL1 dynamics. (a) Steady-state levels of FBPs in wild-type (WT) and $\Delta$ knd1 cells. The expression of Pof1p, Pof9p and Pof10p modified with c-Myc epitopes at the endogenous genomic locus was determined by immunoblotting with Myc antibodies. Blots were reprobed with anti-Skp1p and anti-Cdc2p (PSTAIRE) antibodies as a reference. (b) The first three bars show a quantification of the FBP ratios in $\Delta$ knd1 versus WT cells apparent from the immunoblots in $\mathbf{a}$ as determined by film densitometry. Ratios are from values normalized to Cdc $2 p$. The fourth bar shows the average Skp1p ratio obtained from four independent immunoblot measurements including s.d. The fifth bar shows the $\Delta$ knd1/WT ratio of Skp1p quantified by LC-MS/MS. Skp1p was immunopurified independently four times from 1:1 mixtures of ${ }^{15} \mathrm{~N}(\mathrm{WT})$ and ${ }^{14} \mathrm{~N}(\Delta$ knd 7$)$ labelled cells. The average ratio and the s.d. were plotted. The sixth bar shows the average ratio of all proteins identified in the Skp1p purification. The ratio of these background proteins was close to 1.0 with a narrow s.d., supporting the significance of the $\sim 1.5$-fold increase in Skp1p levels in $\Delta$ knd 1 cells. (c) Schematic outline of the ${ }^{15} \mathrm{~N}$ pulse labelling strategy. Cells maintained in routine ${ }^{14} \mathrm{~N}$ media were shifted to ${ }^{15} \mathrm{~N}$ media for 120 or 240 min before the preparation of cell lysate, Cul1p IP and analysis by LC-MS/MS. (d) Relative fractions of newly synthesized FBPs and COP9 signalosome (CSN) subunits associated with Cul1p in wild-type and $\Delta k n d 1$ cells. Data represent averages and s.d. values of three independent experiments (see Supplementary Data 4).

F-box motif of Pof15p, confirming that it is not an indirect effect of overexpressing Pof15p (Supplementary Fig. S3). Importantly, the displacement of Pof10p-Myc from Cullp by the competing Pof $15 p$ was drastically reduced in the absence of Knd1p even though Pof15p accumulated more readily upon overexpression in $\Delta k n d 1$ cells (Fig. 3a). The inefficiency of Pof $15 p$ in competing off Pof10p from Cullp in the absence of Knd1p suggests that Pof15p is compromised in assembling into a CRL1 complex. Indeed, Pof15p expressed at low, non-competing levels in wild-type and $\Delta k n d 1$ cells was less efficiently recruited into a complex with Cullp when Knd1p was missing (Fig. 3b). Thus, CAND1/Knd1p appears to assist in CRL1 assembly by destabilizing pre-existing Cullp-FBP interactions thereby making Cullp available for engagement in new CRL1 complexes.

CAND1 displaces FBPs from Cul1p in vitro. To further test this idea, we asked whether recombinant CAND1 could displace FBPs from Cullp. Cullp complexes were immunopurified and incubated with recombinant human CAND1 (Supplementary Fig.
S4a) for 30 min followed by measuring the amount of Pof10pMyc retained in the complex. In a reciprocal experiment, Pof10pMyc complexes were purified and incubated with CAND1. In both experiments, CAND1 led to an apparent release of Pof10p from Cullp (Fig. 4a). The same activity of CAND1 was observed for Poflp-Myc complexes (Supplementary Fig. S4b). A kinetic experiment showed that the FBP releasing activity of CAND1 depended on its ability to bind Cullp, as fully neddylated Cullp complexes isolated from $\Delta k n d 1 \Delta c s n 5$ cells were largely resistant to CAND1-mediated FBP release (Fig. 4b). The minor release of Pof 10p that occurred after 100 min of incubation with CAND1 is consistent with the recent observation that neddylated Cullp retains a minimal ability to interact with CAND1 (ref. 30). Lack of the release activity of CAND1/Knd1p may thus cause the increased stability of Cullp-FBP interactions observed in vivo.

CAND1/Knd1p is required for efficient substrate degradation. Our data suggested an important role for CAND1/Knd1p in organizing the cellular CRL1 repertoire such as to optimize 
a
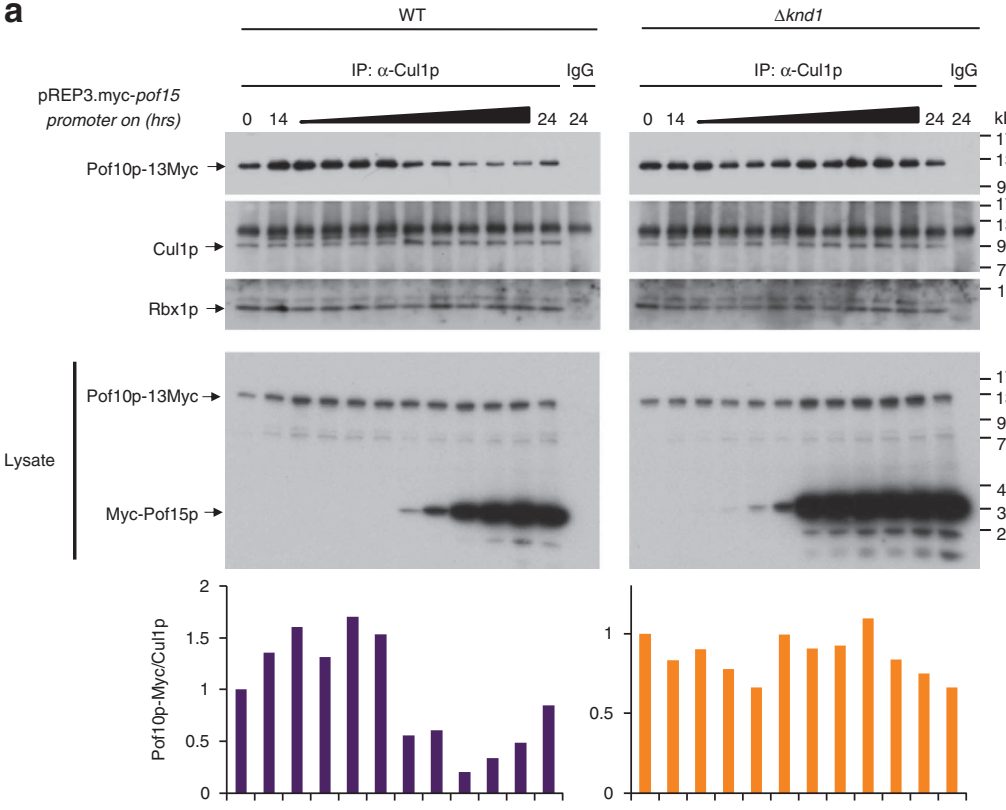

b

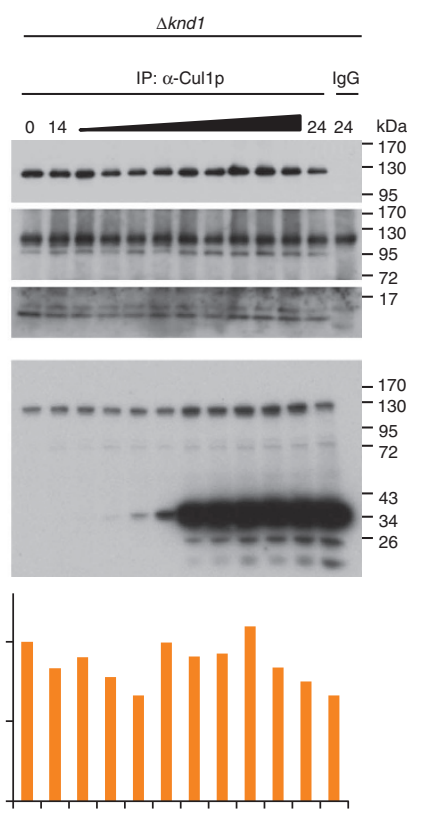

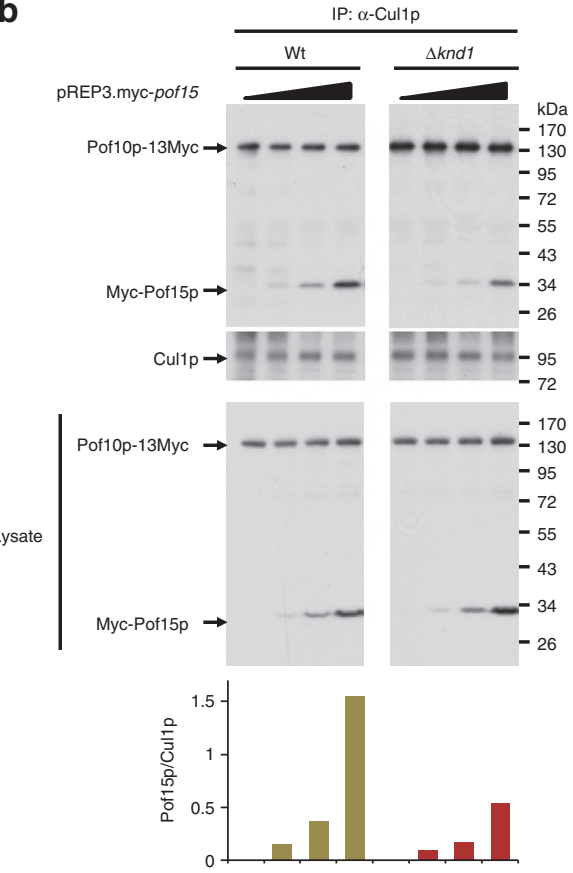

Figure 3 | CRL1 complexes are stabilized in the absence of Knd1p. (a) Myc epitope-tagged Pof15p was expressed from a pREP3 plasmid containing an inducible promoter in strains that harbour endogenously tagged Pof10p-Myc. The strains were constructed in two backgrounds, wild-type (WT) and $\Delta k n d 1$. Myc-Pof15p expression was switched on by the removal of thiamine for $14-24 \mathrm{~h}$ as indicated. CRL1 complexes were immunoprecipitated with anti-Cul1p antibodies and monitored for the levels of co-precipitated Pof10p-Myc and Myc-Pof15p. As a specificity control, the 24-h lysate was used for immunoprecipitation with rabbit IgG. The level of Pof10p bound to Cul1p was quantified and plotted in a bar chart. The bottom panel shows total lysates. (b) Same experiment as in a, but with Myc-Pof15p expressed at low, non-competing levels. The level of Pof15p bound to Cul1p was quantified and plotted in a bar chart.

substrate degradation. To test this, we assessed the impact of Knd1p on the degradation of the CRL1 ${ }^{\text {Pof3p }}$ substrate Ams2p (ref. 31). Similar to what we describe for CRL1 complexes containing Pof5p, Pof $11 \mathrm{p}$ and Pof $15 \mathrm{p}$ in Fig. 1b, the level of CRL1 ${ }^{\text {Pof } 3 \mathrm{p}}$ is $\sim 50 \%$ diminished in $\Delta k n d 1$ cells $^{12}$. Consistent with this downregulation, we found a $\sim 2$-fold upregulation of the steady-state levels of Ams $2 p$ in $\Delta k n d 1$ cells, which coincided with an increase in half-life (Fig. 5a). Similar to $\Delta p o f 3$ cells, which accumulate Ams2p (ref. 24), we found $\Delta k n d 1$ cells to be sensitive to the genotoxic replication inhibitor hydroxyurea (Fig. 5b). CAND1/Knd1p-mediated maintenance of the CRL1 landscape, therefore, appears critical for substrate degradation and normal physiology.

\section{Discussion}

Our data obtained by complete genetic ablation of CAND1 suggest a novel function of CAND1/Knd1p in controlling the in vivo dynamic exchange of FBPs from Cullp. We propose that lack of this activity 'freezes' FBP exchange. Therefore, in the presence of stable but limiting amounts of Cul1p, those CRL1 complexes that are formed by FBPs that have relatively higher intrinsic ratios of on-rate/off-rate for Cullp would out-compete those formed by FBPs with lower ratios. In addition, the degradation rates of individual FBPs may vary and, in the absence of CAND1/Knd1p, FBPs that turn over more quickly would become progressively displaced by those that are more stable thus leading to the observed imbalances. These imbalances may augment the degradation of substrates of CRL1 complexes that accumulate in the absence of CAND1/Knd1p while at the same time curtailing the destruction of substrates of those CRL1 ligases that are depleted. The $\mathrm{CRL1}^{\text {Pof } 3 p}$ substrate Ams $2 p$ is one such example. This interpretation can reconcile conflicting reports of simultaneous positive as well as negative effects of CAND1 depletion on CRL-mediated substrate degradation in vivo $^{32}$.

The proposition that CAND1/Knd1p functions transiently as a FBP exchange factor can rationalize our previous observation that only a very small fraction of Knd1p is in a stable complex with Cullp (ref. 12), a finding that was subsequently confirmed in human cells ${ }^{27}$. This finding combined with the data on CRL dynamics and our demonstration that CAND1 can displace FBPs from Cul1p in vitro strongly suggest that CAND1 drives a cycle of continuous and rapid FBP recruitment and displacement which we dubbed the 'CAND1 cycle'12. This cycle would provide the dynamicity that assures comprehensive sampling of the steadystate FBP repertoire. Our data, therefore, qualify the conclusion of a previous study that CRL network organization is driven by the abundance of FBPs but not by cycles of CAND1 binding and release $^{27}$. Rather, we argue that CAND1/Knd1p accounts for the activity that assures that the CRL1 repertoire is a reflection of the steady-state abundance of FBPs. These considerations also resolve the surprising observation that the CRL1 repertoire is not greatly disturbed when CUL1 neddylation is pharmacologically inhibited by MLN492427,33 because CAND1-mediated equilibration of FBPs would continue to operate-and in fact would be predicted to be more efficient-in the absence of neddylation.

From our studies, the general principle arises that the problem of substrate receptor competition that is inherent to the vast combinatorial architecture of CRLs is solved through the coordinated interplay of the CAND1 cycle with the neddylation/deneddylation cycle. For a given molecule of CUL1, the CAND1-mediated continuous substrate receptor exchange cycle would be interrupted only upon neddylation. As substrate can 

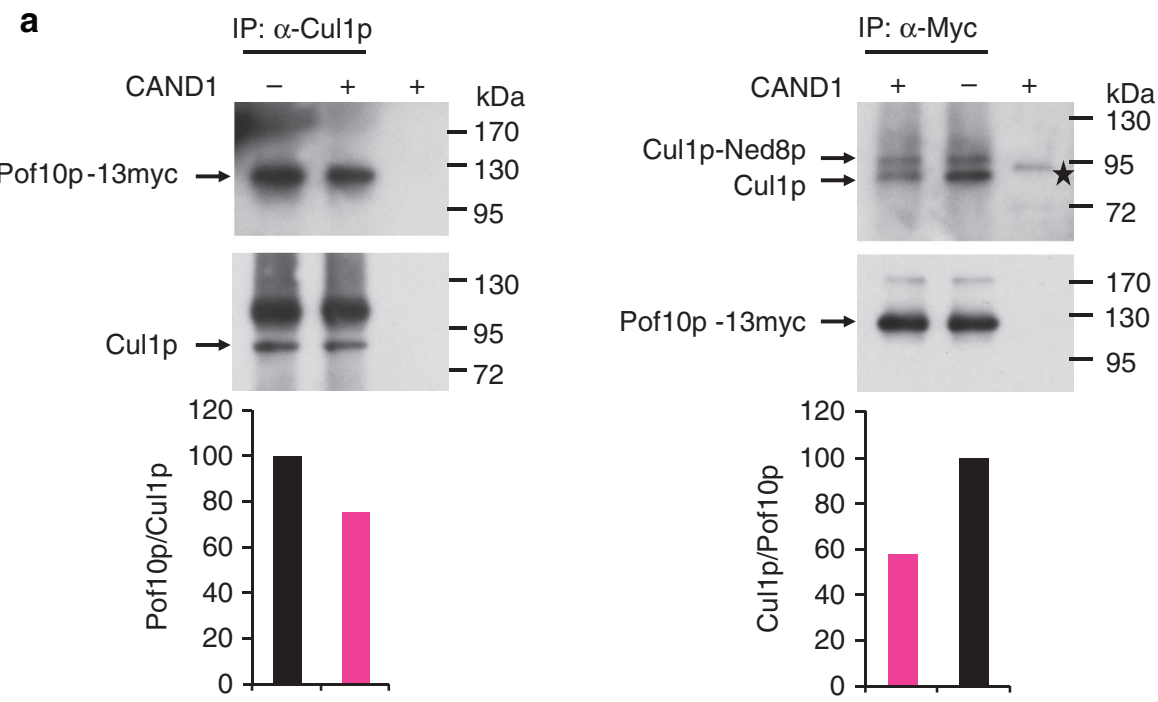

b
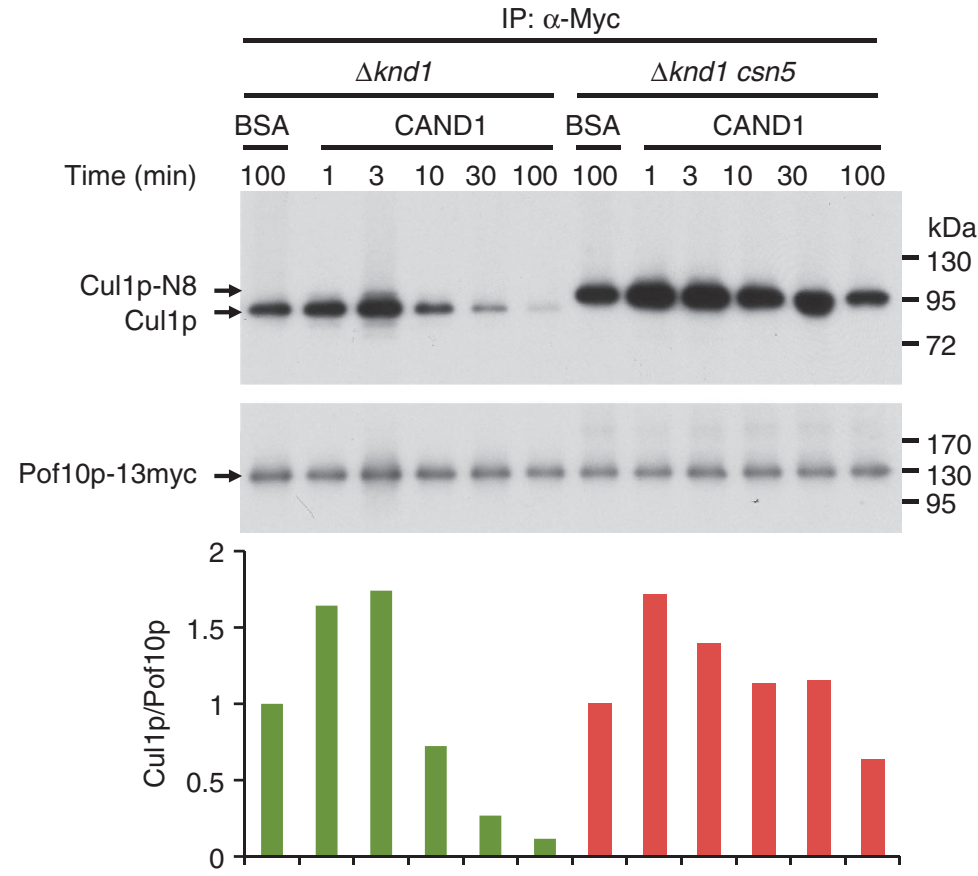

Figure 4 | CAND1 displaces F-box proteins from Cul1p. (a) Cul1p- and Pof10p-associated protein complexes were immunopurified from $\Delta$ knd1 cells and incubated with $1 \mu \mathrm{g}$ of recombinant His-tagged human CAND1 for $30 \mathrm{~min}$. One microgram of bovine serum albumin was used as control instead of CAND1. The complexes were analysed for the levels of Cul1p and Pof10p-Myc by immunoblotting with Cul1p and Myc antibodies, and signals were quantified. The asterisk denotes a band present in the CAND1 preparation that weakly crossreacts with the $\alpha$-Cul1p antiserum (possibly insect cell CUL1). (b) Pof10p-Myc complexes were isolated from $\Delta k n d 1$ and $\Delta k n d 1 \operatorname{csn} 5$ cells as in (a) and incubated for the indicated times with $1 \mu g$ BSA or CAND1 as indicated. The complexes were analysed for the levels of Cul1p and Pof10p-Myc by immunoblotting with Cul1p and Myc antibodies, and signals were quantified.

stimulate cullin neddylation ${ }^{19,30,34}$, CRLs containing receptors fitting a particular substrate that is present at a certain point in time would be selectively removed from the CAND1 cycle and thus activated. Upon substrate consumption, substrate receptors likely succumb to autocatalytic destruction or are recycled ${ }^{11,35}$. COP9 signalosome-mediated deneddylation would re-enter these complexes into the CAND1 cycle. In this scenario, which is consistent with in vitro data ${ }^{18,19}$ as well as our study on in vivo CRL dynamics, CAND1 would function as a substrate receptor exchange factor that accelerates the approach of the unneddylated CRL1 repertoire to equilibrium. Interestingly, CAND1 was originally identified as a factor that stimulates the integration of RNA polymerase II/TFIIF into the TFIID-TFIIB-DNA-complex ${ }^{36}$, raising the intriguing possibility that CAND1 may also promote the dynamics of other large protein complexes that require the same high degree of dynamicity as CRLs.

\section{Methods}

${ }^{15} \mathrm{~N}$ stable isotope labelling and immunopurification of CRL1. The yeast strains used in this study are summarized in Supplementary Table S2. For stable isotope labelling, cells lacking any nutritional markers were grown at $30^{\circ} \mathrm{C}$ for 10 generations on Edinburgh minimal medium (EMM) plates containing either ammonium- ${ }^{14} \mathrm{~N}$ chloride (strain SDW542/2, cul1-13myc:kan knd1::ura4) or 
a

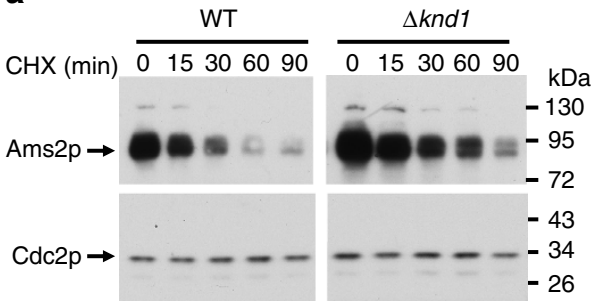

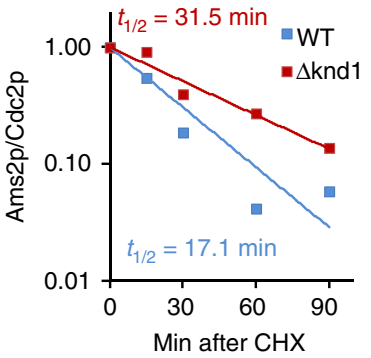

b

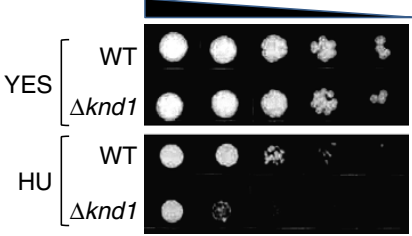

Figure 5 | Knd1p is required for efficient CRL1-mediated substrate degradation. (a) Wild-type and $\Delta$ knd1 cells were incubated with $100 \mu g \mathrm{ml}^{-1}$ cycloheximide (CHX) for the indicated times and Ams2p levels were determined by immunoblotting relative to the loading control Cdc2p. The graph on the right shows a quantification of the results. Exponential trend lines were fitted through the data points in Excel, and the resulting equations were used to calculate half-lives. (b) Hydroxyurea sensitivity of $\Delta k n d 1$ cells. Fivefold serial dilutions of wild-type and $\Delta k n d 1$ cells were spotted onto media with (bottom) or without (top) $10 \mathrm{mM}$ hydroxyurea.

ammonium- ${ }^{15} \mathrm{~N}$ chloride (strain SDW542/1, cul1-13myc:kan) as the nitrogen sources. ${ }^{15} \mathrm{~N}$ labelling efficiency was at least $98 \%$ as determined by mass spectrometry. For immunopurification of CRL1 complexes, cells were grown to optical density $(\mathrm{OD})_{600 \mathrm{~nm}} \sim 1.0$ in $1,000 \mathrm{ml}$ EMM supplemented with the respective light or heavy ammonium chloride. Cells were harvested by centrifugation and washed with STOP buffer $\left(150 \mathrm{mM} \mathrm{NaCl}, 10 \mathrm{mM}\right.$ EDTA, $\left.50 \mathrm{mM} \mathrm{NaF}, 1 \mathrm{mM} \mathrm{NaN}_{3}\right)$ once and flash frozen in liquid nitrogen. Equal amounts of wild-type and $\Delta k n d 1$ mutant cells were mixed and disrupted by bead lysis in $14 \mathrm{ml}$ immunoprecipitation buffer (25 mM Tris- $\mathrm{HCl} \mathrm{pH} \mathrm{7.5,} 50 \mathrm{mM} \mathrm{NaCl}, 0.1 \%$ Triton X100, $1 \mathrm{mM}$ phenylmethyl sulphonyl fluoride (PMSF), $5 \mu \mathrm{g} \mathrm{ml}^{-1}$ aprotinin, $10 \mu \mathrm{g} \mathrm{ml}^{-1}$ leupeptin, $10 \mu \mathrm{g} \mathrm{ml}^{-1}$ pepstatin). Cell lysates were cleared by centrifugation at 18,000 r.p.m. and subjected to immunoprecipitation with $300 \mu \mathrm{l}$ pre-equilibrated anti-c-Myc agarose (Clontech, cat no. 631208) for $2 \mathrm{~h}$. Beads were washed and eluted with $200 \mu \mathrm{l}$ of $100 \mathrm{mM}$ glycine, $\mathrm{pH} 2.0$ and neutralized with $20 \mu \mathrm{l}$ of $2 \mathrm{M}$ Tris- $\mathrm{HCl}, \mathrm{pH}$ 9.0. Five percent of the eluate was loaded onto a gel to estimate the protein amount. The remainder was analysed by LC-MS/MS.

Pulse labelling with ${ }^{15} \mathbf{N}$. Wild-type (SDW542/1) and $\Delta k n d 1$ mutant cells (SDW542/2) were grown to $\mathrm{OD}_{600 \mathrm{~nm}} \sim 1.0$ in $1,000 \mathrm{ml}$ EMM containing ammonium- ${ }^{14} \mathrm{~N}$ chloride (light medium) at $30^{\circ} \mathrm{C}$. Cells were harvested by centrifugation and transferred to $1,000 \mathrm{ml}$ of EMM containing ammonium- ${ }^{15} \mathrm{~N}$ chloride (heavy media). After labelling periods of 120 or $240 \mathrm{~min}$, cells were harvested by centrifugation and washed with STOP buffer once and frozen in liquid nitrogen. Cell lysis and affinity purification of CRL1 complexes was as described above.

One-dimensional LC-MS/MS. Immunopurified protein complexes were first reduced and alkylated using Tris (2-carboxyethyl) phosphine hydrochloride and iodoacetamide, then digested using Trypsin as previously described in detail ${ }^{37}$. After desalting, the peptides were analysed by reverse-phase LC-MS/MS, using a Michrom Paradigm high-performance liquid chromatography with a Magic C18 column and a LTQ-Orbitrap XL mass spectrometer (Thermo-Fisher). The peptides were separated on a 120 -min gradient of $10-30 \%$ buffer B (100\% acetonitrile/0.1\% formic acid). Tandem mass spectrometry (MS/MS) spectra were collected during the LC-MS runs. Each scan was set to acquire a full MS scan, followed by MS/MS scans on the four most intense ions from the preceding MS scan. To maximize coverage, each IP sample was analysed by LC-MS/MS three times (technical replicates).

Data analysis and protein quantification. Both protein identification and quantification of isotopically labelled peptides/proteins were performed by IP2, a comprehensive programme package including SEQUEST, ProLuCID, DTASelect and Census (Integrated Proteomics Applications Inc., San Diego, CA, USA). First, MS1 and MS2 files were extracted by RawExtract (The Scripps Research Institute, La Jolla, CA, USA) from the raw LC-MS/MS spectra obtained on the LTQ-Orbitrap XL. Then a Swissprot S. pombe protein database was uploaded into IP2, which automatically generated a reverse decoy protein database. The MS1 and MS2 files of the three technical replicates of each sample were uploaded to IP2 server, and the combined MS1 and MS2 files were searched using ProLuCID and the decoy database. Differential modification included $16 \mathrm{Da}$ on methionine for oxidation. The identification results from ProLuCID were filtered and organized by DTASelect. The DTASelect parameters were carefully set so that the false-positive rate of protein identification was below $1.5 \%$. The heavy and light spectral counts were obtained for each protein after ProLuCID/DTASelect analysis. Finally the quantification analysis was selected for ${ }^{15} \mathrm{~N}$. Protein ratios and s.d. values were calculated by Census (based on peptides with a factor of determination of $\geq 0.5)$. ${ }^{15} \mathrm{~N}$ enrichment of each sample was calculated by IP2 as well and found to be at least $98 \%$. Protein quantifications with high s.d. values for individual peptides or low peptide numbers were manually examined, and outliers were removed if necessary. To adjust for slight differences in ${ }^{15} \mathrm{~N}$ labelling efficiency in short-term experiments, the protein ratios in the pulse labelling samples were normalized to the average ratio of all proteins quantified in the corresponding sample from wild-type cells. Average protein ratios were averaged over multiple replicates and s.d. values and $P$-values were calculated. To obtain robust data sets, quantifications with a relative s.d. $>35 \%$ were excluded from the final data.

AQUA of CRL1 components by LC-MS/MS. Based on our LC-MS/MS analysis of tryptic digests, we selected peptides TFFETNFIENTK, ELADDDVIWHR, TGVSLQSFQFR and LSFLDEYSLR as AQUA standard peptides for Cullp, Poflp, Pof10p and Pof15p, respectively. The AQUA peptides were purchased from Thermo-Fisher and Sigma-Aldrich, with stable isotopes $\left({ }^{13} \mathrm{C},{ }^{15} \mathrm{~N}\right)$ in the C-terminal lysine or arginine. As the AQUA reference peptides are isotopically distinct from their corresponding endogenous ${ }^{14} \mathrm{~N}$ and ${ }^{15} \mathrm{~N}$ peptides, we can quantify both 'light' and 'heavy' proteins in a single analysis of immunopurified CRL1 complexes isolated from ${ }^{15} \mathrm{~N}$ or ${ }^{14} \mathrm{~N}$-labelled cells. An LTQ-Orbitrap method was developed that performs selected ion monitoring followed by MS/MS confirmation of the peptides. A quantity of $100 \mathrm{fmol}$ of the standard peptides was spiked into the digests of CRL1 complexes immunopurified from a 1:1 mixture of wild-type and $\Delta k n d 1$ cell lysate. The peptides were separated by the same $120 \mathrm{~min}$ gradient LC method on the same LC-LTQ Orbitrap XL system as used for peptide identification. During the AQUA analysis, selected ion monitoring spectra were first collected in the $630-760 \mathrm{~m} / \mathrm{z}$ range with 60,000 Fourier transform mass spectrometry (FTMS) resolution, followed by MS/MS scans of the nine peptide ions with known $\mathrm{m} / \mathrm{z}$. Peak integrations were obtained through the application of extracted ion chromatograms over 10-p.p.m. mass intervals on Qual Browser (Thermo-Fisher). No contaminating peptides were found to coelute with the four peptides chosen for Cullp, Poflp, Pof10p and Pof15p. The analysis was repeated five times. Raw data for a representative AQUA experiment are shown in Supplementary Fig. 5.

Hydroxyurea sensitivity assay. Fivefold serial dilutions of wild-type (SDW542/1) and $\Delta k n d 1$ mutant (SDW542/2) cells were spotted onto control YES plates or plates containing $10 \mathrm{mM}$ hydroxyurea. Plates were incubated at $30^{\circ} \mathrm{C}$ for 5 days.

FBP competition experiment. The pof 15 (SPAPB1A10.14) gene was cloned into the vector pREP3-6His.Myc, which drives the expression of N-terminal 6His-Myctagged protein from the thiamine repressible $n m t 1$ promoter. A Pof $15 \mathrm{p}$ mutant lacking residues 34-40 (LPVEVID) within the F-box motif was generated in the same expression vector. The plasmid were confirmed by sequencing and transformed into wild-type and $\Delta k n d 1$ mutant cells harbouring a Myc-tagged allele of pof 10 at the endogenous genomic locus (pof10-13myc). The expression of pof 15 was induced by removal of thiamine from the culture medium for increasing periods of time followed by preparation of cell lysates by bead lysis in immunoprecipitation buffer ( $25 \mathrm{mM}$ Tris-HCl pH 7.5, $50 \mathrm{mM} \mathrm{NaCl}, 0.1 \%$ Triton X100, $1 \mathrm{mM}$ PMSF, $5 \mu \mathrm{g} \mathrm{ml}^{-1}$ aprotinin, $10 \mu \mathrm{g} \mathrm{ml}^{-1}$ leupeptin, $10 \mu \mathrm{g} \mathrm{ml}^{-1}$ pepstatin). Lysates were subjected to immunoprecipitation with rabbit anti-Cullp antibody ${ }^{38}$, followed by collection of immunocomplexes on protein A Sepharose beads (Rockland, cat no. PA50-00-0005). The immunoprecipitated complexes were analysed by immunoblotting. Myc-tagged FBPs were detected with a monoclonal anti-Myc antibody (9E10, cat. no. 13-2500, Invitrogen). 
Expression and purification of recombinant human CAND1. The CAND1 isoform 1 open reading frame was PCR amplified from the MegaMan Human Transcriptome library (Agilent) with $3^{\prime}$-sequences encoding a hexahistidine tag immediately $5^{\prime}$ of the stop codon and used to generate a recombinant baculovirus using the FastBac system (Invitrogen). After $48 \mathrm{~h}$ of infecting Hi5 insect cell cultures (10e8 cells) at a multiplicity of infection $>10$, CAND1 was purified by Ni-NTA chromatography and desalted into $25 \mathrm{mM}$ HEPES pH 7.6, $100 \mathrm{mM} \mathrm{NaCl}$ and $10 \%$ glycerol.

FBP displacement assay with recombinant CAND1. Cells harbouring endogenous Pof10p modified with Myc epitopes were grown to an $\mathrm{OD}_{600 \mathrm{~nm}}$ of $\sim 1.0$ in $200 \mathrm{ml}$ of YES. Cell lysates were prepared by beads lysis in immunoprecipitation buffer $(25 \mathrm{mM}$ Tris-HCl pH 7.5, $50 \mathrm{mM} \mathrm{NaCl}$ 0.1\% Triton X100, $1 \mathrm{mM} \mathrm{PMSF}, 5 \mu \mathrm{g} \mathrm{ml}^{-1}$ aprotinin, $10 \mu \mathrm{g} \mathrm{ml}^{-1}$ leupeptin, $10 \mu \mathrm{g} \mathrm{ml}^{-1}$ pepstatin). Pof10p was immunopurified on preequilibrated anti-c-Myc agarose beads (Clontech, cat. no. 631208). In a reciprocal experiment, Cullp complexes were immunoprecipitated with Cullp antibodies. The complexes were washed and divided into two aliquots. One microgram of recombinant His-tagged human CAND1 expressed in and purified from insect cells was added to one aliquot and incubated at $30^{\circ} \mathrm{C}$ for $30 \mathrm{~min}$. One microgram of bovine serum albumin was used as control instead of CAND1. After washing, the immunoprecipitated complexes were analysed by immunoblot. Myc-tagged protein was detected with monoclonal anti-c-Myc antibody (9E10, cat. no. 13-2500, Invitrogen), and Cullp with rabbit anti-Cullp antibody.

Immunoprecipitation and immunoblotting of CRL1 complexes. Cell lysates for immunoprecipitation of Myc-tagged FBPs were prepared as described in a buffer containing $50 \mathrm{mM}$ Tris, $\mathrm{pH} 7.4,50 \mathrm{mM} \mathrm{NaCl}$ and $0.5 \%$ Triton X100 (ref. 12). Lysates were cleared by centrifugation, and proteins were precipitated with the respective antisera (anti-Myc, anti-Rbxlp). Immunocomplexes were collected by binding to protein A beads, washed and analysed by immunoblotting. Affinitypurified rabbit antisera against Cullp, Skplp and Rbxlp were described previously ${ }^{38,39}$. Ams $2 p$ antisera were kindly provided by Y. Takayama ${ }^{31}$. For loading controls Cdc2p (PSTAIRE, Santa Cruz) antibodies were used at a dilution of $1: 500$

\section{References}

1. Petroski, M. D. \& Deshaies, R. J. Function and regulation of cullin-RING ubiquitin ligases. Nat. Rev. Mol. Cell. Biol. 6, 9-20 (2005).

2. Bosu, D. R. \& Kipreos, E. T. Cullin-RING ubiquitin ligases: global regulation and activation cycles. Cell Div. 3, 7 (2008).

3. Hua, Z. \& Vierstra, R. D. The cullin-RING ubiquitin-protein ligases. Annu. Rev. Plant Biol. 62, 299-334 (2010).

4. Wolf, D. A., Zhou, C. \& Wee, S. The COP9 signalosome: an assembly and maintenance platform for cullin ubiquitin ligases? Nat. Cell Biol. 5, 1029-1033 (2003).

5. Duda, D. et al. Structural insights into NEDD8 activation of cullin-RING ligases: conformational control of conjugation. Cell 134, 995-1006 (2008).

6. Saha, A. \& Deshaies, R. Multimodal activation of the ubiquitin ligase SCF by Nedd8 conjugation. Mol. Cell. 32, 21-31 (2008).

7. Lyapina, S. et al. Promotion of NEDD8-CUL1 conjugate cleavage by COP9 signalosome. Science 292, 1382 (2001).

8. Schwechheimer, C. et al. Interactions of the COP9 signalosome with the E3 ubiquitin ligase SCFTIR1 in mediating auxin response. Science 292, 1379 (2001).

9. Zhou, C. et al. The fission yeast COP9/signalosome is involved in cullin modification by ubiquitin-related Ned8p. BMC Biochem. 2, 7 (2001).

10. Zhou, C. et al. Fission yeast COP9/signalosome suppresses cullin activity through recruitment of the deubiquitylating enzyme Ubp12p. Mol. Cell 11, 927-938 (2003).

11. Cope, G. A. \& Deshaies, R. J. Targeted silencing of Jab1/Csn5 in human cells downregulates SCF activity through reduction of F-box protein levels. BMC Biochem. 7, 1 (2006).

12. Schmidt, M. W., McQuary, P. R., Wee, S., Hofmann, K. \& Wolf, D. A. F-Boxdirected CRL complex assembly and regulation by the CSN and CAND1. Mol. Cell 35, 586-597 (2009).

13. Liu, J., Furukawa, M., Matsumoto, T. \& Xiong, Y. NEDD8 modification of CUL1 dissociates p120CAND1, an inhibitor of CUL1-SKP1 binding and SCF ligases. Mol. Cell 10, 1511-1518 (2002).

14. Zheng, J. et al. CAND1 binds to unneddylated CUL1 and regulates the formation of SCF ubiquitin E3 ligase complex. Mol. Cell 10, 1519-1526 (2002).

15. Hwang, J.-W., Min, K.-W., Tamura, T. \& Yoon, J.-B. TIP120A associates with unneddylated cullin 1 and regulates its neddylation. FEBS Lett. 541, 102-108 (2003).

16. Min, K.-W. TIP120A associates with cullins and modulates ubiquitin ligase activity. J. Biol. Chem. 278, 15905-15910 (2003).

17. Oshikawa, K. et al. Preferential interaction of TIP120A with Cul1 that is not modified by NEDD8 and not associated with Skp1. Biochem. Biophys. Res. Commun. 303, 1209-1216 (2003).
18. Siergiejuk, E. et al. Cullin neddylation and substrate-adaptors counteract SCF inhibition by the CAND1-like protein Lag2 in Saccharomyces cerevisiae. EMBO J 28, 3845-3856 (2009).

19. Bornstein, G., Ganoth, D. \& Hershko, A. Regulation of neddylation and deneddylation of cullin1 in SCFSkp2 ubiquitin ligase by F-box protein and substrate. Proc. Natl Acad. Sci. 103, 11515-11520 (2006).

20. Chuang, H., Zhang, W. \& Gray, W. M. Arabidopsis ETA2, an apparent ortholog of the human cullin-interacting protein CAND1, is required for auxin responses mediated by the SCFTIR1 ubiquitin ligase. Plant Cell 16, 1883 (2004).

21. Feng, S. et al. Arabidopsis CAND1, an unmodified CUL1-interacting protein, is involved in multiple developmental pathways controlled by ubiquitin/ proteasome-mediated protein degradation. Plant Cell 16, 1870 (2004).

22. Bosu, D. R. et al. C. elegans CAND-1 regulates cullin neddylation, cell proliferation and morphogenesis in specific tissues. Dev. Biol. 346, 113-126 (2010).

23. Helmstaedt, K. et al. Recruitment of the inhibitor CAND1 to the cullin substrate adaptor site mediates interaction to the neddylation site. Mol. Biol. Cell 22, 153-164 (2011).

24. Katayama, S., Kitamura, K., Lehmann, A., Nikaido, O. \& Toda, T. Fission yeast F-Box protein Pof3 is required for genome integrity and telomere function. Mol. Biol. Cell 13, 211-224 (2002).

25. Lo, S. C. \& Hannink, M. CAND1-mediated substrate adaptor recycling is required for efficient repression of Nrf2 by Keap1. Mol. Cell. Biol. 26, 1235 (2006).

26. Zhang, W. et al. Genetic analysis of CAND1-CUL1 interactions in Arabidopsis supports a role for CAND1-mediated cycling of the SCFTIR1 complex. Proc. Natl Acad. Sci. 105, 8470-8375 (2008).

27. Bennett, E. J., Rush, J., Gygi, S. P. \& Harper, J. W. Dynamics of cullin-RING ubiquitin ligase network revealed by systematic quantitative proteomics. Cell 143, 951-965 (2010).

28. Washburn, M. P., Ulaszek, R., Deciu, C., Schieltz, D. M. \& Yates, III J. R. Analysis of quantitative proteomic data generated via multidimensional protein identification technology. Anal. Chem. 74, 1650-1657 (2002).

29. Gerber, S. A., Rush, J., Stemman, O., Kirschner, M. W. \& Gygi, S. P. Absolute quantification of proteins and phosphoproteins from cell lysates by tandem MS Proc. Natl Acad. Sci. USA 100, 6940-6945 (2003).

30. Emberley, E. D., Mosadeghi, R. \& Deshaies, R. J. Deconjugation of Nedd8 from Cull is directly regulated by Skp1-Fbox and substrate, and CSN inhibits deneddylated SCF by a non-catalytic mechanism. J. Biol. Chem. 287, 29679-29689 (2012).

31. Takayama, Y. et al. Hsk1- and SCFPof3-dependent proteolysis of S. pombe Ams2 ensures histone homeostasis and centromere function. Dev. Cell 18, 385-396 (2010).

32. Kim, S.-H., Kim, H.-J., Kim, S. \& Yim, J. Drosophila CAND1 regulates cullin3dependent E3 ligases by affecting the neddylation of Cullin 3 and by controlling the stability of Cullin3 and adaptor protein. Dev. Biol. 346, 247-257 (2010).

33. Lee, J. E. et al. The steady-state repertoire of human SCF ubiquitin ligase complexes does not require ongoing Nedd8 conjugation. Mol. Cell. Proteomics 10, M110.006460 (2011)

34. Chew, E.-H. \& Hagen, T. Substrate-mediated regulation of Cullin neddylation. J. Biol. Chem. 282, 17032-17040 (2007).

35. Wee, S., Geyer, R. K., Toda, T. \& Wolf, D. A. CSN facilitates cullin-RING ubiquitin ligase function by counteracting autocatalytic adapter instability. Nat. Cell Biol. 7, 387-391 (2005).

36. Makino, Y. et al. TATA-binding protein-interacting protein 120, TIP120, stimulates three classes of eukaryotic transcription via a unique mechanism. Mol. Cell. Biol 19, 7951-7960 (1999).

37. Brill, L. M., Motamedchaboki, K., Wu, S. \& Wolf, D. A. Comprehensive proteomic analysis of Schizosaccharomyces pombe by two-dimensional HPLCtandem mass spectrometry. Methods 48, 311-319 (2009).

38. Seibert, V. et al. Combinatorial diversity of fission yeast SCF ubiquitin ligases by homo- and heterooligomeric assemblies of the F-box proteins Poplp and Pop2p. BMC Biochem. 3, 22 (2002).

39. Geyer, R., Wee, S., Anderson, S., Yates, J. \& Wolf, D. A. BTB/POZ domain proteins are putative substrate adaptors for cullin 3 ubiquitin ligases. Mol. Cell 12, 783-790 (2003).

\section{Acknowledgements}

We thank Y. Takayama for Ams2p antibodies and Z. Ronai for review of the manuscript. We also thank R. Deshaies and T. Kurz for sharing results prior to publication. This work was supported by NIH grants GM59780, P20 CA132386-03 and P50 GM085764-03.

\section{Author contributions}

S.W. prepared yeast strains and all samples for MS analysis, contributed to MS data analysis and performed immunoblots. W. Z. performed LC-MS/MS and MS data analysis. T.N. prepared yeast strains and performed immunoblot analyses and the hydroxyurea sensitivity experiment. J.I.T and M.D.P. expressed and purified human 
CAND1. D.A.W. conceived the study, directed the experimental design, performed immunoprecipitation experiments, assisted with data analysis and drafted the manuscript.

\section{Additional information}

Supplementary Information accompanies this paper at http://www.nature.com/ naturecommunications
Competing financial interests: The authors declare no competing financial interests.

Reprints and permission information is available online at http://npg.nature.com/ reprintsandpermissions/

How to cite this article: $\mathrm{Wu}, \mathrm{S}$. et al. CAND1 controls in vivo dynamics of the Cullin 1-RING ubiquitin ligase repertoire. Nat. Commun. 4:1642 doi: 10.1038/ncomms2636 (2013). 